\title{
An automated one-way avoidance box for the rat
}

S. S. TENEN ${ }^{1}$

CHAS. PFIZER \& CO., INC.

An automated one-way avoidance box was described and the results of a standardized procedure were presented. In using this apparatus the importance of escape platform time was demonstrated.

In studying the discriminated avoidance response and especially its acquisition it is generally more desirable to use automated equipment. Since the leverpress response is subject to considerable difficulty (Meyer et al, 1960) some variation of the shuttlebox has often been used. One of the advantages of this bi-directional procedure is that a series of automatically programmed trials can be given without repeated handling of the animals. However, sometimes certain theoretical or experimental variables require that a one-way avoidance procedure be used (Vanderwolf, 1963; Kunitomi et al, 1964; Theios \& Dunaway, 1964; Kenyon \& Krieckhaus, 1965; Moyer \& Korn, 1966). Not only are the practical problems of running a oneway procedure by hand sometimes prohibitive but the repeated handling of the animals might introduce variables that influence the results (e.g., Zucker, 1965). Also, a one-way procedure is superior to a two-way one when rapid acquisition of a relatively stable avoidance response is required.

Figure 1 depicts an automated one-way avoidance box that has been in use about one year now. The principle is simply a sliding wall (A) moved by a motor (C) driven rack and pinion which exposes an escape platform (B). The sliding wall is programmed to recede simultaneously with the onset of an auditory (G) conditioned stimulus. A $60 \mathrm{rpm}$ reversible motor (e.g., Bodine, 6.6 in./oz torque, type KYC-23RB; price \$29) and a pinion gear that has a diameter of $9 / 16$ in. (12 teeth) will fully expose the platform (5 in. depth) in about $2.5 \mathrm{sec}$. This travel rate is fast enough to permit an avoidance response to be made in about 1.0 sec. and slow enough to provide a gentle return of the animal to the grid floor. The photoelectric cells (E) are not disrupted by the movement of the sliding wall (1/4 in. clear Plexiglas) when travelling at this rate. The animal's interruption of the photoelectric cells is used to terminate the auditory stimulus and operate programming and recording equipment. After a specified period of time on the escape platform the rat is returned to the grid floor to await another trial.

The mechanically smooth and gentle dispossession provided by the sliding wall does not appear to produce any physical discomfort to the rat. Some limited experience in using this box with rats with wires attached to chronically implanted (brain) electrodes has also proven successful. The rats did not get

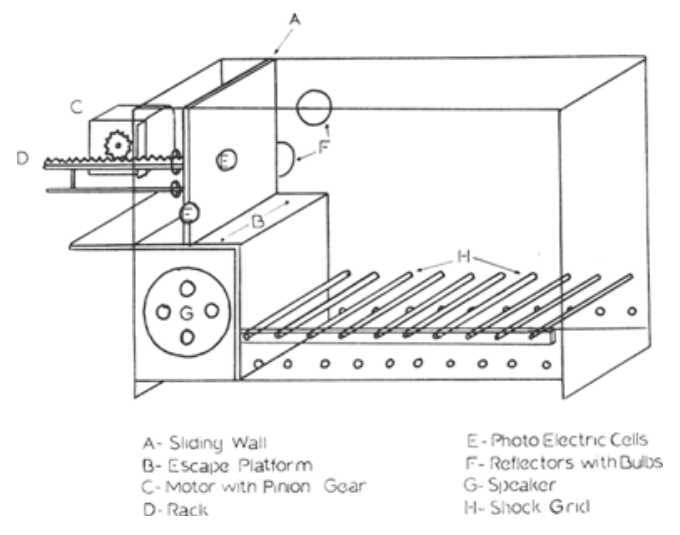

Fig. 1. An automated one-way avoidance box.

entangled with the wires, nor did the wires hamper their performance of the response.

The bars (H) of the grid floor are rigidly attached to an external piece of plastic so that they can be quickly removed from the upper holes and placed in the lower ones in order to vary the jumping distance. The box is made of $1 / 4 \mathrm{in}$. Plexiglas and the overall dimensions are: 8 in. wide, 14 in. long (with sliding wall closed) and the grid floor is 4 in. (or 6 in.) below the escape platform and 11 in. (or 13 in.) from the top.

The sliding wall is mounted so that it moves without resting on the platform floor. Control over its back and forth movements is accomplished with two simple "stops" (Fig. 2) and a push-button Microswitch (B2R88-A2). The "stops" first terminate the current to the motor by depressing the microswitch and are tapered to provide a more positive halt of the wall. Once the microswitch and "stops" are adjusted they appear to provide reliable performance.

One of the procedures that we have been using was designed for moderately rapid avoidance acquisition. The rats ( $N=118$, male albino, Charles River CD strain)

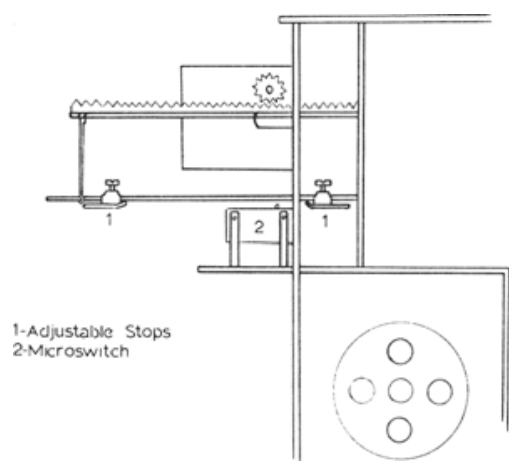

Fig. 2. Control of sliding wall. 
were given a standard 30 trials in which the shock intensity was about $0.5 \mathrm{ma}$, the CS-shock interval was 10 sec., the escape platform time was $15 \mathrm{sec}$, the intertrial interval (on the grid floor) was $45 \mathrm{sec}$., (if the rat failed to escape the shock for $30 \mathrm{sec}$. the trial was automatically terminated and the next trial started 45 sec. later). The average number of avoidance responses made was about 20 and $74 \%$ of the group made at least 18 . Only three rats failed to make any avoidance responses at all, two of which failed even to make escape responses.

In addition to the usual variables (e.g., shock intensity, CS-US interval, etc.) that can affect avoidance acquisition it appears that escape-platform time (amount of reinforcement ?) is yet another one. For example, two groups of rats $(\mathrm{N}=10 \mathrm{each})$ were trained with the above procedure except one group was allowed only $4 \mathrm{sec}$. escape platform time instead of $15 \mathrm{sec}$. The mean number of avoidances made by the 15 sec. group $(M=$ $22.1)$ was significantly $(p<.01)$ greater than that made by the 4 sec. group ( $M=15.8)$. In using this apparatus, then, escape platform time would have to be considered when comparing results of different procedures.

\section{References}

Kenyon, J., \& Krieckhaus, E. E. Decrements in one-way avoidance learning following septal lesions in rats. Psychon. Sci., 1965. 3, 113-114,

Kunitomi, E., Shikano, T., \& Imada, H. Avoidance learning in shuttling and non-shuttling situations with and without a barrier. Jap. Psychol. Res., 1964, 6, 129-135.

Meyer, D. R., Cho, C., \& Weseman, A. On the problems of conditioning discriminated lever-press avoidance response. Psychol. Pev., 1960, 67, 224-228.

Moyer, K. E., \& Korn, J. H. Effect of UCS intensity on the acquisition and extinction of a one-way avoidance response. Psychon. Sci., 1966, 4, 121-122.

Theios, J., \& Dunaway, J. E. One-way vs. shuttle avoidance conditioning. Psychon. Sci., 1964, 1, 251-252.

Vanderwolf, C. H. Improved shuttle box performance following electroconvulsive shock. J. comp. physiol. Psychol., 1963, 56, 983-986.

Zucker, 1. Comment on Kenyon and Krieckhaus. Psychon. Sci., $1965,3,298$.

\section{Nofe}

1. The author is grateful to $\mathrm{J}$. Dzialo for the drawings and to $\mathrm{L}$. Wood and R. Jarvas for their technical assistance. 